\title{
Improved Patient Satisfaction Following TKA Using Intraoperative Computer Technology to Obtain Accurate Gap Balancing
}

\author{
Austin Smith, MD ${ }^{1}$, Christian Eccles, MD ${ }^{1}$, Samrath Bhimani, MD ${ }^{1}$, Kevin \\ Denehy, $\mathrm{MD}^{2}$, Rohat Bhimani, $\mathrm{BA}^{3}$, Langan Smith, $\mathrm{BS}^{4}$, and Arthur Malkani, \\ $\mathrm{MD}^{2}$ \\ ${ }^{1}$ University of Louisville, Dept. of Orthopaedic Surgery \\ ${ }^{2}$ University of Louisville, Adult Reconstruction Program, KentuckyOne Health \\ ${ }^{3}$ Northwestern University, Feinberg School of Medicine \\ ${ }^{4}$ Jewish Hospital, KentuckyOne Health, Louisville, KY \\ austinsmithf@gmail.com, checcles@gmail.com, sambhimani1@gmail.com, denehy@gmail.com, \\ rahool.bhimani@gmail.com, langanssmith@kentuckyonehealth.org, arthur.malkani@louisville.edu
}

\begin{abstract}
Aims:

Approximately $20 \%$ of patients are dissatisfied with their total knee arthroplasty (TKA). Purpose of this study was to determine if patient satisfaction could be improved following TKA using computer technology to obtain the target alignment and precisely balanced gaps.
\end{abstract}

Patients \& Methods:

75 consecutive patients undergoing primary robotic-arm assisted TKA (RA-TKA) with real-time intraoperative alignment and gap balancing information were compared with a prospective cohort of 75 consecutive patients undergoing primary TKA with manual jig-based instruments during the same time period. There were no differences between groups with age, gender, BMI, and ASA scores. TKA's were performed by a single surgeon using same implant design, anesthesia and surgical protocols. Patient satisfaction survey using Knee Society (KSS) and Likert scoring system was obtained at 1-year follow-up.

Results:

Likert scoring system demonstrated $95 \%$ of patients in the computer technology group were either very satisfied or satisfied versus $75 \%$ in the manual instruments TKA group $(p=0.005)$ at 1 -year follow-up. Second question of the KSS which deals with pain at rest was significantly better in RA- 
TKA group ( $\mathrm{p}=0.04)$. Fifth question which deals with recreational activities was also significantly improved in the RA-TKA group, $p=0.02$. RA-TKA group had a better average overall satisfaction score of 7.1 versus 6.4 in the manual instrument group, $p=0.03$.

Conclusion:

Using intraoperative computer technology to achieve the target alignment with flexion/extension gap balancing to within $1 \mathrm{~mm}$, a significant improvement in patient satisfaction was demonstrated compared to TKA using conventional manual jig-based instruments.

\section{Introduction}

In 2012, the number of total knee arthroplasties (TKA) performed in the US was over 700,000 and projected to increase to 3.5 million by 2030 [1]. Today's health care system continues to place emphasis on patient reported outcomes and satisfaction following TKA. Previous reports show that only $75 \%$ of patients are either satisfied or very satisfied with their TKA at 1-year follow-up [2]. Even with contemporary design TKA implants, approximately one in five (19\%) primary TKA patients were not satisfied with their outcome [3]. There are several reasons for patient dissatisfaction following TKAs, including component malalignment and instability [4]. Malalignment of greater than $3^{\circ}$ has been identified in $32 \%$ of conventional TKA's and can lead to symptoms of pain and instability [5]. Instability due to flexion and extension gap mismatch is also one of the leading etiologies of failure of a primary TKA leading to revision surgery $[6,7]$.

The purpose of this study was to determine if primary TKA performed using computer technology to visualize and balance the flexion and extension gaps within $1 \mathrm{~mm}$ along with robotic assisted surgery to obtain exact bone cuts would lead to improved patient satisfaction compared to manual jig-based instruments.

\section{Materials and Methods}

Consecutive patients undergoing robotic-arm assisted TKA (RA-TKA) with intraoperative computer technology $\left(\mathrm{Mako}^{\mathrm{TM}}\right.$, Stryker, Mahwah, NJ) or traditional jigbased TKA using manual instruments were prospectively studied. Inclusion criteria included patients undergoing primary TKA for osteoarthritis and minimum of 1-year follow-up. 2 patients were excluded due to a periprosthetic joint infection leaving 150 patients available for review. There were 75 patients that underwent primary RATKA and 75 patients that underwent primary conventional TKA. There were no differences between cohorts with respect to age, gender, and BMI. 
Arthroplasties were performed by a single surgeon using the same implant design (Triathlon ${ }^{\mathrm{TM}}$ PS TKA, Stryker, Mahwah, New Jersey). All cases were performed during the same time period using the same surgical technique, anesthesia team and post-operative protocols. In the RA-TKA cohort, preoperative CT scans were utilized to prepare a virtual $3 \mathrm{D}$ pre-operative plan with respect to the desired component position, limb alignment and gap measurements. The goal was to obtain equal gap measurements within $1 \mathrm{~mm}$ between the flexion and extension gaps and the medial and lateral gaps. The goal of the limb alignment was a target within 3 degrees of the mechanical axis. In the conventional TKA cohort, a measured resection technique was performed using intramedullary alignment for the distal femoral cut and an external alignment guide for the proximal tibial cut.

Patients were given the patient satisfaction derived portion of the 2011 Knee Society Scoring System (KSS) to complete independently at 1-year follow-up appointments [8]. KSS function and knee scores were also recorded preoperatively and at 6-week and 1-year follow-up visits. Clinical results and complications were also assessed and compared between the groups; length of stay, operative time and pre and postoperative active range of motion were analyzed.

\section{Results}

At 1-year follow-up, overall patient reported satisfaction reported with a Likert scoring system demonstrated a statistically significant difference with $95 \%$ of patients in the computer technology TKA group being either very satisfied or satisfied versus $75 \%$ in the TKA group $(\mathrm{p}=0.005)$. The second question of the KSS which assesses satisfaction with level of pain while in bed at rest showed a significant difference with the computer technology TKA group having $91 \%$ of patients very satisfied or satisfied versus $75 \%$ in the TKA group $(p=0.04)$. The RA-TKA group had a statistically significant better average total score on the KSS Satisfaction survey of 34.2 versus $31.4(\mathrm{p}=0.046)$ (Table 1).

Both groups had improved KSS scores following surgery. The RA-TKA cohort showed improved postoperative function scores vs the conventional TKA group and there was a statistically significant difference in 1-year function scores with an average score of 78 vs 70 in the RA-TKA and conventional TKA cohorts, respectively $(\mathrm{p}=0.02)$. A summary of the Knee Society Scores, LOS and operative time is seen in Table 2. 


\section{Discussion}

Use of advanced technology in TKA to help achieve the desired surgical goals of the target alignment and balanced gaps has demonstrated improved outcomes and can benefit the surgeon intraoperatively to achieve the desired goals with greater accuracy than manual instruments. The use of manual jig based instruments does not provide real time information on femoral component alignment in the coronal, axial, and sagittal planes nor does it provide tibial component position with the same degree of accuracy compared to the use of intraoperative computer technology. Both measured resection techniques and gap balancing techniques for performing primary TKA do not provide the exact numerical information on component alignment nor the exact gap measurement. With the ability to achieve gap balancing within $1 \mathrm{~mm}$ between the medial and lateral gaps and also the flexion and extension gaps, we were able to demonstrate improved patient satisfaction up to $95 \%$ compared to $75 \%$ in the manual jig based group (Figure 1). Gap balancing has been demonstrated to be of paramount importance for successful TKA. However, despite the surgeons' best efforts, the use of manual jig based instruments does not intraoperatively provide the information required for precision balancing compared to the use of computer technology.

The use of computer technology with robotic assisted surgery is becoming more widespread in all surgical fields. With the use of intraoperative computer technology we were able to demonstrate $95 \%$ either satisfied or very satisfied in

patients undergoing TKA compared to $75 \%$ with conventional TKA using manual jig based instruments which is a positive change compared to aforementioned studies in the literature with the use of manual instruments [2,3]. Computer technology for TKA provides real time intraoperative numerical information on implant alignment and gap measurements, not available with manual jig based techniques, to allow for accurate bone cuts, implant placement, and gap balancing. The use of this innovative technology leads to accurate placement of total knee implants to achieve the target alignment and the ability to accurately balance flexion and extension gaps within $1 \mathrm{~mm}$ leading to improved patient satisfaction. 


\section{References}

1. Kurtz SM, Lau E, Ong K, Zhao K, Kelly M, Bozic KJ. Future young patient demand for primary and revision joint replacement: National projections from 2010 to 2030. Clin Orthop Relat Res. 2009;467(10):2606-2612. doi:10.1007/s11999-009-0834-6.

2. Noble PC, Conditt MA, Cook KF, Mathis KB. The John Insall Award: Patient expectations affect satisfaction with total knee arthroplasty. Clin Orthop Relat Res. 2006;452(452):35-43. doi:10.1097/01.blo.0000238825.63648.1e.

3. Bourne RB, Chesworth BM, Davis AM, Mahomed NN, Charron KDJJ. Patient satisfaction after total knee arthroplasty: Who is satisfied and who is not? Clin Orthop Relat Res. 2010;468(1):57-63. doi:10.1007/s11999-009-1119-9.

4. Gunaratne R, Pratt DN, Banda J, Fick DP, Khan RJK, Robertson BW. Patient Dissatisfaction Following Total Knee Arthroplasty: A Systematic Review of the Literature. $J \quad$ Arthroplasty. 2017;32(12):3854-3860. doi:10.1016/j.arth.2017.07.021.

5. Mason JB, Fehring TK, Estok R, Banel D, Fahrbach K. Meta-Analysis of Alignment Outcomes in Computer-Assisted Total Knee Arthroplasty Surgery. J Arthroplasty. 2007;22(8):1097-1106. doi:10.1016/j.arth.2007.08.001.

6. Sharkey PF, Hozack WJ, Rothman RH, Shastri S, Jacoby SM. Why are total knee arthroplasties failing today? Clin Orthop Relat Res. 2002;(404):7-13. doi:10.1097/01.blo.0000036002.13841.32.

7. Fehring TK, Valadie AL. Knee instability after total knee arthroplasty. Clin Orthop Relat Res. 1994;(299):157-162.

8. Noble PC, Scuderi GR, Brekke AC, et al. Development of a New Knee Society Scoring System. Clin Orthop Relat Res. 2012;470(1):20-32. doi:10.1007/s11999-011-2152-z. analysis. Surg. 2018;16(4):237-244. doi:https://doi.org/10.1016/j.surge.2017.12.003. 


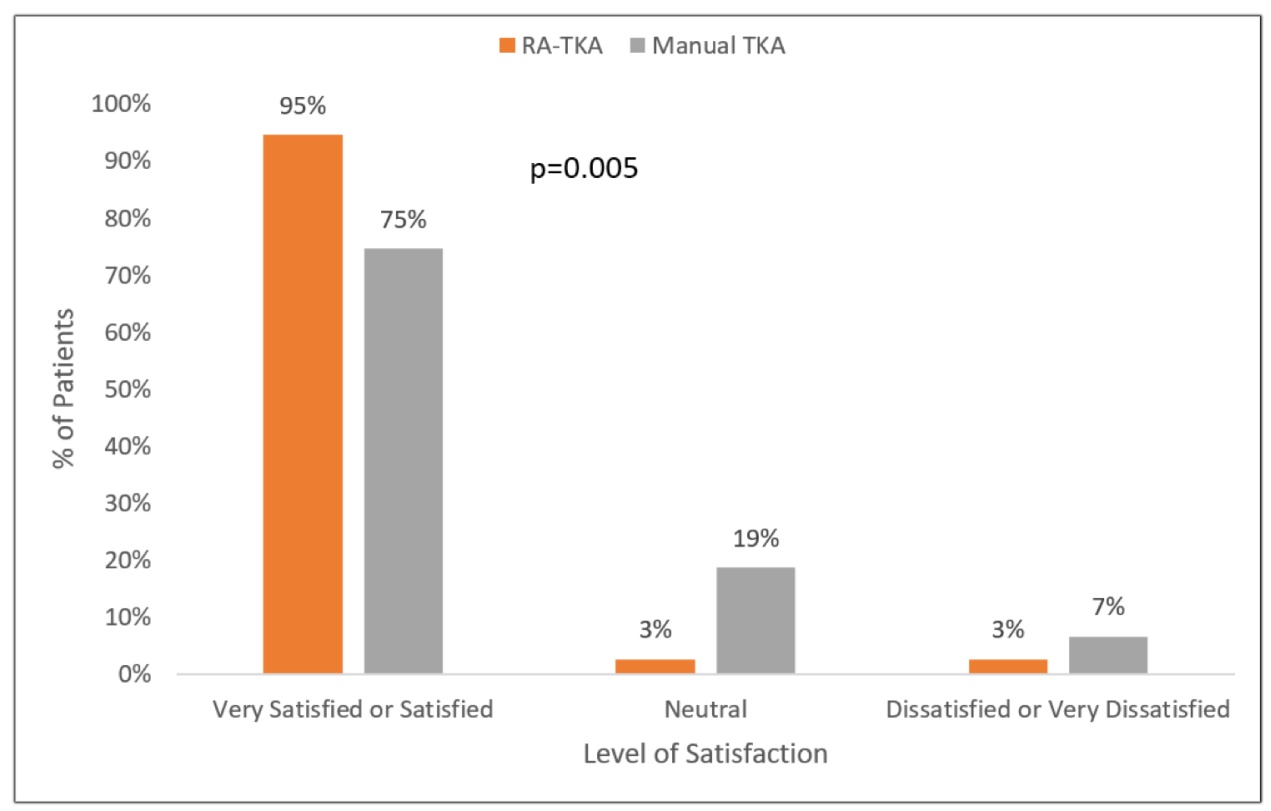

Figure 1: Bar graph demonstrating the distribution of patient satisfaction 1-year postoperatively between the RA-TKA group and the manual instruments TKA group.

Table 1: Comparison of average numerical responses to each question five questions of KSS patient satisfaction section, the total section score, and the overall Likert question between the RA-TKA group and the manual instruments TKA group.

\begin{tabular}{lcccc}
\hline & RA-TKA & Manual TKA & $\Delta$ & p-value \\
\hline \hline Question 1 & 7.1 & 6.8 & 0.35 & 0.22 \\
Question 2 & 6.9 & 6.3 & 0.59 & 0.07 \\
Question 3 & 6.9 & 6.5 & 0.43 & 0.15 \\
Question 4 & 6.7 & 6.1 & 0.59 & 0.053 \\
Question 5 & 6.5 & 5.7 & 0.85 & 0.02 \\
\hline Total Score & 34.2 & 31.4 & 2.80 & 0.046 \\
\hline Overall Satisfaction Question & 7.1 & 6.4 & 0.69 & 0.03 \\
\hline
\end{tabular}


Table 2: Preoperative and 6-week and 1-year postoperative Knee Society Scores and length of stay (LOS) and operative time compared between the RA-TKA group and the manual TKA group.

\begin{tabular}{lccc}
\hline & RA-TKA & Manual TKA & p-value \\
\hline \hline Operative time (hours, minutes) & $1: 41$ & $1: 27$ & $<0.01$ \\
LOS (days) & 2 & 3 & 0.05 \\
Preop KSS function score & 41 & 41 & 0.99 \\
6 week KSS function score & 62 & 58 & 0.12 \\
1 year KSS function score & 78 & 70 & 0.02 \\
Preop KSS knee score & 40 & 38 & 0.33 \\
6 week KSS knee score & 81 & 77 & 0.12 \\
1 year KSS knee score & 83 & 83 & 0.88 \\
\hline
\end{tabular}

